\title{
THE SECOND DUAL SPACES OF THE SETS OF $\Lambda$-STRONGLY CONVERGENT AND BOUNDED SEQUENCES
}

\section{A. M. JARRAH and E. MALKOWSKY}

(Received 19 November 1998)

\begin{abstract}
We give the second $\beta$-, $\gamma^{-}$, and $f$-duals of the sets $w_{0}^{p}(\Lambda), w_{\infty}^{p}(\Lambda)(0<p<\infty)$, $c_{0}^{p}(\Lambda), c^{p}(\Lambda)$, and $c_{\infty}^{p}(\Lambda)(0<p \leq 1)$ and the second continuous dual spaces of $w_{0}^{p}(\Lambda)$, $c_{0}^{p}(\Lambda)$, and $c^{p}(\Lambda)$ for $0<p \leq 1$. Furthermore, we determine the $\alpha$-duals of $c_{0}^{p}(\Lambda), c^{p}(\Lambda)$, and $c_{\infty}^{p}(\Lambda)$ for $1<p<\infty$.
\end{abstract}

Keywords and phrases. BK spaces, AK spaces, AD spaces, dual spaces.

2000 Mathematics Subject Classification. Primary 40H05, 46A45; Secondary 47B07.

1. Introduction and well-known results. We write $\omega$ for the set of all complex sequences $x=\left(x_{k}\right)_{k=0}^{\infty}, \phi, l_{\infty}, c$ and $c_{0}$ for the sets of all finite, bounded, convergent sequences, and sequences convergent to naught, respectively, further $c s, b s$, and $l_{1}$ for the sets of all convergent, bounded, and absolutely convergent series.

By $e$ and $e^{(n)}\left(n \in \mathbb{N}_{0}\right)$, we denote the sequences such that $e_{k}=1$ for $k=0,1, \ldots$, and $e_{n}^{(n)}=1$ and $e_{k}^{(n)}=0$ for $k \neq n$. For any sequence $x=\left(x_{k}\right)_{k=0}^{\infty}$, let $x^{[n]}=\sum_{k=0}^{n} x_{k} e^{(k)}$ be its $n$-section.

Let $X, Y \subset \omega$ and $z \in \omega$. Then we write

$$
\begin{aligned}
& z^{-1} \times X=\left\{x \in \omega: x z=\left(x_{k} z_{k}\right)_{k=0}^{\infty} \in X\right\}, \\
& M(X, Y)=\bigcap_{x \in X} x^{-1} \times Y=\{a \in \omega: a x \in Y \forall x \in X\}
\end{aligned}
$$

for the multiplier space of $X$ and $Y$. The sets $M\left(X, l_{1}\right), M(X, c s)$, and $M(X, b s)$ are called the $\alpha$-, $\beta$-, and $\gamma$-duals of $X$.

A Fréchet subspace $X$ of $\omega$ is called an FK space if it has continuous coordinates, that is, if convergence in $X$ implies coordinatewise convergence. An FK space $X \supset \phi$ is said to have AK if, for every sequence $x=\left(x_{k}\right)_{k=0}^{\infty} \in X, x^{[n]} \rightarrow x(n \rightarrow \infty)$; and it is said to have AD if $\phi$ is dense in $X$. A BK space is an FK space which is a Banach space.

If $X$ is a $p$-normed space, then we write $X^{*}$ for the set of all continuous linear functionals on $X$, the so-called continuous dual of $X$, with its norm $\|\cdot\|$ is given by

$$
\|f\|=\sup \{|f(x)|:\|x\|=1\} \quad \forall f \in X^{*} .
$$

Let $X \supset \phi$ be an FK space. Then the set $X^{f}=\left\{\left(f\left(e^{(n)}\right)\right)_{n=0}^{\infty}: f \in X^{*}\right\}$ is called the $f$-dual of $X$.

Given any infinite matrix $A=\left(a_{n k}\right)_{n, k=0}^{\infty}$ of complex numbers and any sequence $x \in \omega$, let $A_{n}(x)=\sum_{k=0}^{\infty} a_{n k} x_{k}(n=0,1, \ldots)$, and let $A(x)=\left(A_{n}(x)\right)_{n=0}^{\infty}$ provided the 
series converge, and $X_{A}=\{x \in \omega: A(x) \in X\}$. If $0<p<\infty$, then we write $|x|^{p}=$ $\left(\left|x_{k}\right|^{p}\right)_{k=0}^{\infty}$ and $X_{[A]^{p}}=\left\{x \in \omega: A\left(|x|^{p}\right) \in X\right\}$.

Let $0<p<\infty$ and $\mu=\left(\mu_{n}\right)_{n=0}^{\infty}$ be a nondecreasing sequence of positive integers tending to infinity, throughout. We define the matrices $\Delta$ and $M$ by

$$
\begin{aligned}
\Delta_{n k} & = \begin{cases}1 & (k=n), \\
-1 & (k=n-1), \\
0 & \text { (otherwise) },\end{cases} \\
M_{n k} & =\left\{\begin{array}{ll}
\frac{1}{\mu_{n}^{p}} & (0 \leq k \leq n) \\
0 & (k>n)
\end{array} \quad(n=0,1, \ldots)\right.
\end{aligned}
$$

and use the convention that any symbol with a negative subscript has the value zero.

The sets

$$
\begin{gathered}
w_{0}^{p}(\mu)=\left(c_{0}\right)_{[M]^{p}}, \quad w_{\infty}^{p}(\mu)=\left(l_{\infty}\right)_{[M]^{p}}, \\
c_{0}^{p}(\mu)=(\mu)^{-1} \times\left(w_{0}^{p}(\mu)\right)_{\Delta}, \quad c_{\infty}^{p}(\mu)=(\mu)^{-1} \times\left(w_{\infty}^{p}(\mu)\right)_{\Delta}, \quad c^{p}(\mu)=c_{0}^{p}(\mu) \oplus e
\end{gathered}
$$

were studied in [1], and their first duals were given there. If $p=1$, then we omit the index $p$, i.e., we write $w_{0}(\mu)=w_{0}^{1}(\mu)$, etc.

Following the notation introduced in [3], we say that a nondecreasing sequence $\Lambda=$ $\left(\lambda_{n}\right)_{n=0}^{\infty}$ of positive reals tending to infinity is exponentially bounded if there are reals $s$ and $t$ with $0<s \leq t<1$ such that for some subsequence $\left(\lambda_{n(v)}\right)_{v=0}^{\infty}$ of $\Lambda$, we have

$$
s \leq \frac{\lambda_{n(v)}}{\lambda_{n(v+1)}} \leq t \quad \forall v=0,1, \ldots
$$

such a subsequence $\left(\lambda_{n(v)}\right)_{v=0}^{\infty}$ is called an associated subsequence.

If $(n(v))_{v=0}^{\infty}$ is a strictly increasing sequence of nonnegative integers, then we write $K^{\langle v\rangle}$ for the set of all integers $k$ with $n(v) \leq k \leq n(v+1)-1$, and $\sum_{v}$ and $\max _{v}$ for the sum and maximum taken over all $k$ in $K^{\langle v\rangle}$.

If $X^{p}(\Lambda)$ denotes any of the sets $w_{0}^{p}(\Lambda), w_{\infty}^{p}(\Lambda), c_{0}^{p}(\Lambda), c^{p}(\Lambda)$, or $c_{\infty}^{p}(\Lambda)$, then we write $\tilde{X}^{p}(\Lambda)$ for the respective space with the sections $1 / \lambda_{n}^{p} \sum_{k=0}^{n} \ldots$ replaced by the blocks $1 / \lambda_{n(v+1)}^{p} \sum_{v} \ldots$. Furthermore, we define

$$
\begin{aligned}
& \|x\|_{w_{\infty}^{p}(\Lambda)}= \begin{cases}\sup _{n}\left(\frac{1}{\lambda_{n}^{p}} \sum_{k=0}^{n}\left|x_{k}\right|^{p}\right) & (0<p \leq 1), \\
\sup _{n}\left(\frac{1}{\lambda_{n}^{p}} \sum_{k=0}^{n}\left|x_{k}\right|^{p}\right)^{1 / p} & (1 \leq p<\infty),\end{cases} \\
& \|x\|_{\tilde{w}_{\infty}^{p}(\Lambda)}= \begin{cases}\sup _{v}\left(\frac{1}{\lambda_{n(v+1)}^{p}} \sum_{v}\left|x_{k}\right|^{p}\right) & (0<p \leq 1), \\
\sup _{v}\left(\frac{1}{\lambda_{n(v+1)}^{p}} \sum_{v}\left|x_{k}\right|^{p}\right)^{1 / p} & (1 \leq p<\infty),\end{cases} \\
& \|x\|_{c_{\infty}^{p}(\Lambda)}=\|\Delta(\Lambda x)\|_{w_{\infty}^{p}(\Lambda)},\|x\|_{\tilde{c}_{\infty}^{p}(\Lambda)}=\|\Delta(\Lambda x)\|_{\tilde{w}_{\infty}^{p}(\Lambda)} .
\end{aligned}
$$


2. The second duals of the sets $w_{0}^{p}(\Lambda)$ and $w_{\infty}^{p}(\Lambda)$ for $0<p<\infty$. Let $\Lambda=\left(\lambda_{n}\right)_{n=0}^{\infty}$ be a nondecreasing exponentially bounded sequence of positive reals throughout and let $\left(\lambda_{n(v)}\right)_{v=0}^{\infty}$ be an associated subsequence. We put

$$
\mathscr{W}^{p}(\Lambda)= \begin{cases}\left\{a \in \omega: \sum_{v=0}^{\infty} \lambda_{n(v+1)} \max _{v}\left|a_{k}\right|<\infty\right\} & (0<p \leq 1), \\ \left\{a \in \omega: \sum_{v=0}^{\infty} \lambda_{n(v+1)}\left(\sum_{v}\left|a_{k}\right|^{p}\right)^{1 / p}<\infty\right\} & \left(1<p<\infty, q=\frac{p}{p-1}\right)\end{cases}
$$

and, on $\mathcal{W}^{p}(\Lambda)$,

$$
\|a\|_{\mathscr{W} p(\Lambda)}= \begin{cases}\sum_{v=0}^{\infty} \lambda_{n(v+1)} \max _{v}\left|a_{k}\right| & (0<p \leq 1), \\ \sum_{v=0}^{\infty} \lambda_{n(v+1)}\left(\sum_{v}\left|a_{k}\right|^{p}\right)^{1 / p} & \left(1<p<\infty, q=\frac{p}{p-1}\right) .\end{cases}
$$

In [1, Theorem 2], it was shown that if $X^{p}(\Lambda)=w_{0}^{p}(\Lambda)$ or $X^{p}(\Lambda)=w_{\infty}^{p}(\Lambda)$ and $\dagger$ stands for $\alpha, \beta, \gamma$, or $f$, then $\left(X^{p}(\Lambda)\right)^{\dagger}=W^{p}(\Lambda)$, that the continuous dual $\left(w_{0}^{p}(\Lambda)\right)^{*}$ of $w_{0}^{p}(\Lambda)$ is norm isomorphic to $\mathscr{W}^{p}(\Lambda)$ when $w_{0}^{p}(\Lambda)$ has the norm $\|\cdot\|_{\tilde{w}_{\infty}^{p}(\Lambda)}$, and finally that $\|a\|_{\tilde{w}_{\infty}^{p}(\Lambda)}^{*}=\|a\|_{W^{p}(\Lambda)}$ on $\left(w_{\infty}^{p}(\Lambda)\right)^{\beta}$. Furthermore, ${ }^{W^{p}}(\Lambda)$ is a BK space with AK with $\|\cdot\|_{W^{p}(\Lambda)}$ (cf. [2]). Therefore the following result gives the second duals of the sets $w_{0}^{p}(\Lambda)$ and $w_{\infty}^{p}(\Lambda)$.

THEOREM 2.1. We put $p^{\prime}=\max \{1, p\}$. If $\dagger$ stands for any of the symbols $\alpha, \beta, \gamma$, or $f$, then $\left(W^{p}(\Lambda)\right)^{\dagger}=w_{\infty}^{p^{\prime}}(\Lambda)$ for $0<p<\infty$, and the continuous dual $\left(W^{p}(\Lambda)\right)^{*}$ of $\mathcal{W}^{p}(\Lambda)$ is norm isomorphic to $w_{\infty}^{p^{\prime}}(\Lambda)$ with $\|\cdot\|_{\tilde{w}_{\infty}^{p^{\prime}}}$.

Proof. The statements of the theorem with the exception of those concerning the $\gamma$ - and $f$-duals are well known (cf. [2, Theorems 2, 4, 5, and 6]).

Since $\mathscr{W}^{p}(\Lambda)$ has AK, it follows that $\left(\mathcal{W}^{p}(\Lambda)\right)^{\beta}=\left(\mathscr{W}^{p}(\Lambda)\right)^{f}$ by [4, Theorem 7.2.7(ii), page 106], and so $\left(\mathcal{W}^{p}(\Lambda)\right)^{f}=w_{\infty}^{p^{\prime}}(\Lambda)$. Further $\mathcal{W}^{p}(\Lambda)$ has $\mathrm{AD}$, since it has $\mathrm{AK}$, and so $\left(\mathscr{W}^{p}(\Lambda)\right)^{\beta}=\left(\mathscr{W}^{p}(\Lambda)\right)^{\gamma}$ by [4, Theorem 7.2.7(iii), page 106], hence $\left(\mathscr{W}^{p}(\Lambda)\right)^{\gamma}=w_{\infty}^{p^{\prime}}(\Lambda)$.

3. The $\alpha$-duals of the sets $c_{0}^{p}(\Lambda), c^{p}(\Lambda)$, and $c^{p}(\Lambda)$ for $1<p<\infty$

THEOREM 3.1. We put

$$
\begin{gathered}
\mathscr{C}_{\alpha}^{p}(\Lambda)=\left\{a \in \omega: \sum_{v=0}^{\infty} \lambda_{n(v+1)}\left(\sum_{v}\left(\sum_{k=n}^{\infty} \frac{\left|a_{k}\right|}{\lambda_{k}}\right)^{q}\right)^{1 / q}<\infty\right\} \quad\left(1<p<\infty ; q=\frac{p}{p-1}\right), \\
\|a\|_{\mathscr{C}_{\alpha}^{p}(\Lambda)}=\sum_{v=0}^{\infty} \lambda_{n(v+1)}\left(\sum_{v}\left(\sum_{k=n}^{\infty} \frac{\left|a_{k}\right|}{\lambda_{k}}\right)^{q}\right)^{1 / q} .
\end{gathered}
$$

If $X^{p}(\Lambda)$ denotes any of the sets $c_{0}^{p}(\Lambda), c^{p}(\Lambda)$, and $c_{\infty}^{p}(\Lambda)$, then $\left(X^{p}(\Lambda)\right)^{\alpha}=\mathscr{C}_{\alpha}^{p}(\Lambda)$. Furthermore, $\mathscr{C}_{\alpha}^{p}(\Lambda)$ is a BK space with $\|\cdot\|_{\mathscr{C}_{\alpha}^{p}(\Lambda)}$. 
Proof. First, we assume $a \in \mathscr{C}_{\alpha}^{p}(\Lambda)$, and let $x \in c_{\infty}^{p}(\Lambda)$. Then there is a constant $M$ such that

$$
\left(\sum_{v}\left|(\Delta(\Lambda x))_{n}\right|^{p}\right)^{1 / p} \leq \lambda_{n(v+1)} M \quad \forall v=0,1, \ldots
$$

Putting $R_{n}=\sum_{k=n}^{\infty}\left(\left|a_{k}\right| / \lambda_{k}\right)(n=0,1, \ldots)$ and using Hölder's inequality, we obtain

$$
\begin{aligned}
\sum_{k=0}^{\infty}\left|a_{k} x_{k}\right| & \leq \sum_{v=0}^{\infty} \frac{\left|a_{k}\right|}{\lambda_{k}} \sum_{n=0}^{k}\left|(\Delta(\Lambda x))_{n}\right|=\sum_{n=0}^{\infty}\left|(\Delta(\Lambda x))_{n}\right| \sum_{k=n}^{\infty} \frac{\left|a_{k}\right|}{\lambda_{k}} \\
& =\sum_{v=0}^{\infty} \sum_{v}\left|(\Delta(\Lambda x))_{n}\right| R_{n} \leq M \sum_{v=0}^{\infty} \lambda_{n(v+1)}\left(\sum_{v} R_{n}^{q}\right)^{1 / q} .
\end{aligned}
$$

This shows that $\mathscr{C}_{\alpha}^{p}(\Lambda) \subset\left(c_{\infty}^{p}(\Lambda)\right)^{\alpha}$ and that

$$
\sum_{k=0}^{\infty}\left|a_{k} x_{k}\right| \leq\|a\|_{\mathscr{C}_{\alpha}^{p}(\Lambda)}\|x\|_{\tilde{c}_{\infty}^{p}(\Lambda)} .
$$

Conversely, we assume $a \in c_{0}^{p}(\Lambda)$. We define the maps $f_{a}^{(m)}: c_{0}^{p}(\Lambda) \rightarrow \mathbb{R}$ by $f_{a}^{(m)}(x)=$ $\sum_{k=0}^{m}\left|a_{k} x_{k}\right|(x \in X)$. Then $\left(f_{a}^{(m)}\right)_{m=0}^{\infty}$ is a sequence of seminorms on $c_{0}^{p}(\Lambda)$ which are continuous, since $c_{0}^{p}(\Lambda)$ is a BK space by [1, Theorem 1]. Further, $f_{a}^{(m)}(x) \leq$ $\sum_{k=0}^{\infty}\left|a_{k} x_{k}\right|=M(x)<\infty$ for all $m \in \mathbb{N}_{0}$ and for all $x \in X$. By the uniform boundedness principle, there is a constant $M$ such that

$$
\sum_{k=0}^{\infty}\left|a_{k} x_{k}\right| \leq M \quad \forall x \in c_{0}^{p}(\Lambda) \text { with }\|x\|_{\tilde{c}_{\infty}^{p}(\Lambda)} \leq 1 .
$$

Since $a \in\left(c_{0}^{p}(\Lambda)\right)^{\alpha}$ and $1 / \Lambda=\left(1 / \lambda_{k}\right)_{k=0}^{\infty} \in c_{0}^{p}(\Lambda)$, the numbers $R_{n}$ are defined for all $n$. We put

$$
S_{\mu}=\sum_{l=n(\mu)}^{n(\mu+1)-1} R_{l}^{q} \quad \forall \mu=0,1, \ldots
$$

Let $v(m) \in \mathbb{N}_{0}$ be given. We define the sequence $x^{v(m)}$ by

$$
x_{n}^{\nu(m)}= \begin{cases}\frac{1}{\lambda_{n}}\left(\sum_{\mu=0}^{v-1} \lambda_{n(\mu+1)} S_{\mu}^{-1 / p} \sum_{k=n(\mu)}^{n(\mu+1)-1} R_{k}^{q-1}+\lambda_{n(v+1)} S_{v}^{-1 / p} \sum_{k=n(v)}^{n} R_{k}^{q-1}\right) \\ \frac{1}{\lambda_{n}} \sum_{\mu=0}^{v(m)} \lambda_{n(\mu+1)} S_{\mu}^{-1 / p} \sum_{k=n(\mu)}^{n(\mu+1)-1} R_{k}^{q-1} & (n \geq n(v(m)+1)) .\end{cases}
$$

Then

$$
\begin{aligned}
\left(\Delta\left(\Lambda x^{v(m)}\right)\right)_{n} & = \begin{cases}\lambda_{n(v+1)} S_{v}^{-1 / p} R_{n}^{q-1} & \left(n \in N^{\langle v\rangle} ; v=0,1, \ldots, v(m)\right), \\
0 & \left(n \in N^{\langle v\rangle} ; v \geq v(m)+1\right),\end{cases} \\
\sum_{v}\left|\left(\Delta\left(\Lambda x^{v(m)}\right)\right)_{n}\right| & = \begin{cases}\lambda_{n(v+1)}^{p} S_{v}^{-1} \sum_{v} R_{n}^{q}=\lambda_{n(v+1)}^{p} & (0 \leq v \leq v(m)), \\
0 & (v \geq v(m+1)) .\end{cases}
\end{aligned}
$$


Thus $x^{\nu(m)} \in c_{0}^{p}(\Lambda)$ and $\left\|x^{\nu(m)}\right\|_{\tilde{c}_{\infty}^{p}(\Lambda)}=1$. Now, by (3.5) and (3.8) and since $x_{k}^{\nu(m)} \geq 0$ for all $k=0,1, \ldots$,

$$
\begin{aligned}
\sum_{v=0}^{v(m)} \lambda_{n(v+1)}\left(\sum_{v} R_{n}^{q}\right)^{1 / q} & =\sum_{v=0}^{v(m)} \lambda_{n(v+1)}\left(\sum_{v} R_{n}^{q}\right) S_{v}^{-1 / p}=\sum_{v=0}^{v(m)} \sum_{v}\left(\lambda_{n(v+1)} S_{v}^{-1 / p} R_{n}^{q-1}\right) R_{n} \\
& =\sum_{v=0}^{v(m)} \sum_{v}\left|\left(\Delta\left(\Lambda x^{v(m)}\right)\right)_{n}\right| R_{n} \leq \sum_{n=0}^{\infty}\left|\left(\Delta\left(\Lambda x^{v(m)}\right)\right)_{n}\right| \sum_{k=n}^{\infty} \frac{\left|a_{k}\right|}{\lambda_{k}} \\
& =\sum_{k=0}^{\infty} \frac{\left|a_{k}\right|}{\lambda_{k}}\left|\sum_{n=0}^{k}\left(\Delta\left(\Lambda x^{v(m)}\right)\right)_{n}\right|=\sum_{k=0}^{\infty} \frac{\left|a_{k}\right|}{\lambda_{k}} \lambda_{k}\left|x_{k}^{v(m)}\right| \\
& =\sum_{k=0}^{\infty}\left|a_{k}\right|\left|x_{k}^{v(m)}\right| \leq M .
\end{aligned}
$$

Since $v(m) \in \mathbb{N}_{0}$ was arbitrary, we have

$$
\sum_{v=0}^{\infty} \lambda_{n(v+1)}\left(\sum_{v} R_{n}^{q}\right)^{1 / q} \leq \sum_{k=0}^{\infty}\left|a_{k} x_{k}\right|<\infty,
$$

that is, $a \in \mathscr{C}_{\alpha}^{p}(\Lambda)$.

Therefore we have shown $\left(c_{\infty}^{p}(\Lambda)\right)^{\alpha}=\left(c_{0}^{p}(\Lambda)\right)^{\alpha}=\mathscr{C}_{\alpha}^{p}(\Lambda)$. Since $c_{0}^{p}(\Lambda) \subset c^{p}(\Lambda) \subset$ $c_{\infty}^{p}(\Lambda)$ for $1<p<\infty$ (cf. [1, Lemma 1(b)]), we also have $\left(c^{p}(\Lambda)\right)^{\alpha}=\mathscr{C}_{\alpha}^{p}(\Lambda)$.

Finally, $\mathscr{C}_{\alpha}^{p}(\Lambda)$ is a BK space with $\|\cdot\|_{\mathscr{C}_{\alpha}^{p}(\Lambda)}$ by [4, Theorem 4.3.15, page 64], (3.4), and (3.10).

4. The second duals of the sets $c_{0}^{p}(\Lambda), c^{p}(\Lambda)$, and $c_{\infty}^{p}(\Lambda)$ for $0<p \leq 1$. We put

$$
\begin{gathered}
\mathscr{C}(\Lambda)=\left\{a \in \omega: \sum_{v=0}^{\infty} \lambda_{n(v+1)} \max _{v}\left|\sum_{k=n}^{\infty} \frac{a_{k}}{\lambda_{k}}\right|<\infty\right\}, \\
\|a\|_{\mathscr{C}(\Lambda)}=\sum_{v=0}^{\infty} \lambda_{n(v+1)} \max _{v}\left|\sum_{k=n}^{\infty} \frac{a_{k}}{\lambda_{k}}\right| .
\end{gathered}
$$

In [1, Theorem 4], it was shown that if $X^{p}(\Lambda)$ is any of the sets $c_{0}^{p}(\Lambda)$ or $c_{\infty}^{p}(\Lambda)$ and † stands for any of the symbols $\beta, \gamma$, or $f$, then $\left(X^{p}(\Lambda)\right)^{\dagger}=\mathscr{C}(\Lambda)$ and that this also holds when $X^{p}(\Lambda)=c(\Lambda)$ or $X^{p}(\Lambda)=c^{p}(\Lambda)$ for $0<p<1$ whenever

$$
\sup _{n} \frac{1}{\mu_{n}^{p}} \sum_{k=0}^{n}\left|(\Delta(\mu x))_{k}\right|^{p}<\infty ;
$$

otherwise $\left(c^{p}(\Lambda)\right)^{\beta}=\mathscr{C}(\Lambda) \cap c s$ and $\left(c^{p}(\Lambda)\right)^{\gamma}=\mathscr{C}(\Lambda) \cap b s$. Furthermore, it was shown that the continuous dual $\left(c_{0}^{p}(\Lambda)\right)^{*}$ of $c_{0}^{p}(\Lambda)$ is norm isomorphic to $\mathscr{C}(\Lambda)$ when $c_{0}^{p}(\Lambda)$ has the $p$-norm $\|\cdot\|_{\tilde{c}_{\infty}^{p}(\Lambda)}$ and $\|a\|_{\tilde{c}_{\infty}^{p}(\Lambda)}^{*}=\|a\|_{\mathscr{C}(\Lambda)}$ on $c_{\infty}^{p}(\Lambda)$. Finally, that $f \in c^{*}(\Lambda)$ if and only if $f(x)=l_{\chi_{f}}+\sum_{n=0}^{\infty} a_{n} x_{n}$ for all $x \in c(\Lambda)$ where $a \in \mathscr{C}(\Lambda), l \in \mathbb{C}$ with $x-l e \in c_{0}(\Lambda)$ and $\chi_{f}=f(e)-\sum_{n=0}^{\infty} a_{n}$, and that $\|f\|$ is equivalent to $\left|\chi_{f}\right|+\|a\|_{\mathscr{C}(\Lambda)}$; if condition (4.2) is satisfied, then this also holds for $c^{p}(\Lambda)(0<p<1)$.

Therefore the following result gives the second duals. 
THEOREM 4.1. (a) The space $\mathscr{C}(\Lambda)$ with $\|\cdot\|_{\mathscr{C}(\Lambda)}$ is a BK space with $A K$.

(b) The set $c_{\infty}(\Lambda)$ is $\beta$ perfect, that is, $c_{\infty}^{\beta \beta}(\Lambda)=c_{\infty}(\Lambda)$. Further $\|a\|_{\mathscr{C}(\Lambda)}^{*}=\|a\|_{\tilde{c}_{\infty}(\Lambda)}$ for all $a \in \mathscr{C}^{\beta}(\Lambda)$.

(c) Finally, $\mathscr{C}^{f}(\Lambda)=\mathscr{C}^{\gamma}(\Lambda)=\mathscr{C}^{\beta}(\Lambda)$.

Proof. We apply Abel's summation by parts. If $a \in c s$, then we write $R(a)$ for the sequence with $R_{n}(a)=\sum_{k=n}^{\infty} a_{k}(n=0,1, \ldots)$. Then

$$
\sum_{n=0}^{m-1} a_{n} y_{n}=\sum_{n=0}^{m} R_{n}(a)(\Delta y)_{n}-R_{m}(a) y_{m} \quad \forall m=0,1, \ldots
$$

(a) The space $\mathscr{W}(\Lambda)$ is a BK space with $\|\cdot\|_{\mathscr{W}(\Lambda)}$ (cf. [2, Theorem 2]). Further, the matrix $A$ defined by $a_{n k}=1 / \lambda_{k}$ for $k \geq n$ and $a_{n k}=0$ for $0 \leq n-1(n=0,1, \ldots)$ is one-to-one, and $x=A(y) \in \mathscr{C}(\Lambda)$ if and only if $y \in \mathscr{W}(\Lambda)$. So, by [4, Theorem 4.3.2, page 61], $\mathscr{C}(\Lambda)$ is a BK space with $\|x\|_{\mathscr{C}(\Lambda)}=\|A(y)\|_{\mathscr{W}(\Lambda)}$. Now, we show that $\mathscr{C}(\Lambda)$ has AK. First, we observe that $\phi \subset \mathscr{C}(\Lambda)$, since $\mathscr{C}(\Lambda)$ is the $\beta$-dual of a sequence space. Now, let $x \in \mathscr{C}(\Lambda)$ and let $\varepsilon>0$. For each $m \in \mathbb{N}_{0}$, let $v_{m}$ denote the uniquely determined integer for which $m \in N^{\left\langle v_{m}\right\rangle}$. We choose $m_{0} \in \mathbb{N}_{0}$ such that

$$
\sum_{v=v_{m}}^{\infty} \lambda_{n(v+1)} \max _{v}\left|R_{n}(x / \Lambda)\right|<\varepsilon \quad \forall m \geq m_{0} .
$$

Let $m \geq m_{0}$. Since the sequence $\Lambda=\left(\lambda_{n}\right)_{n=0}^{\infty}$ is exponentially bounded, there is $t \in$ $(0,1)$ such that, by $(1.5)$,

$$
\begin{aligned}
\left\|x-x^{[m]}\right\|_{\mathscr{C}(\Lambda)} & =\sum_{v=0}^{\infty} \lambda_{n(v+1)} \max _{v}\left|R_{n}\left(\left(x-x^{[m]}\right) / \Lambda\right)\right| \\
& \leq \sum_{v=0}^{v_{m}-1} \lambda_{n(v+1)}\left|R_{m+1}(x / \Lambda)\right|+\sum_{v=v_{m}}^{\infty} \lambda_{n(v+1)} \max _{v}\left|R_{n}(x / \Lambda)\right| \\
& <\varepsilon+\sum_{v=0}^{v_{m}-1} \frac{\lambda_{n(v+1)}}{\lambda_{n\left(v_{m}+1\right)}} \lambda_{n\left(v_{m}+1\right)} \max _{v_{m}}\left|R_{n}(x / \Lambda)\right| \\
& <\varepsilon+\varepsilon \sum_{v=0}^{v_{m}-1} t^{v_{m}-v}<\varepsilon \frac{1}{1-t} .
\end{aligned}
$$

This shows that $\mathscr{C}(\Lambda)$ has AK.

(b) First, we show that $\mathscr{C}^{\beta}(\Lambda)=c_{\infty}(\Lambda)$.

For any $X \subset \omega, X \subset X^{\beta \beta}$ by [4, Theorem 7.1.2, page 105]. So we have to show $c_{\infty}(\Lambda) \subset$ $\mathscr{C}^{\beta}(\Lambda)$ by $[1$, Theorem 4$]$.

Let $a \in \mathscr{C}^{\beta}(\Lambda)$. We define $f_{a}: \mathscr{C}(\Lambda) \rightarrow \mathbb{C}$ by $f_{a}(x)=\sum_{k=0}^{\infty} a_{k} x_{k}$ for all $x \in \mathscr{C}(\Lambda)$. Then $f_{a} \in \mathscr{C}^{*}(\Lambda)$ by [4, Theorem 7.2.9, page 107], and so

$$
\left|f_{a}(x)\right| \leq\left\|f_{a}\right\|\|x\|_{\mathscr{C}(\Lambda)}<\infty \quad \forall x \in \mathscr{b}(\Lambda)
$$


Let $m \in \mathbb{N}_{0}$ be given and $v_{m}$ the uniquely determined integer such that $m \in N^{\left\langle v_{m}\right\rangle}$. Since $\Lambda$ is exponentially bounded, there are $s, t \in(0,1)$ such that, by (1.5),

$$
\begin{aligned}
\left\|e^{(m)}\right\|_{\mathscr{C}(\Lambda)} & =\sum_{v=0}^{\infty} \lambda_{n(v+1)} \max _{v}\left|R_{n}\left(\frac{e^{(m)}}{\Lambda_{k}}\right)\right|=\sum_{v=0}^{v_{m}} \frac{\lambda_{n(v+1)}}{\lambda_{m}} \\
& \leq \sum_{v=0}^{v_{m}} \frac{\lambda_{n(v+1)}}{\lambda_{n\left(v_{m}+1\right)}} \frac{\lambda_{n\left(v_{m}+1\right)}}{\lambda_{n\left(v_{m}\right)}} \leq \frac{1}{s} \sum_{v=0}^{v_{m}} t^{v_{m}-v} \leq \frac{1}{s(1-t)}<\infty .
\end{aligned}
$$

Now (4.6) implies

$$
\left|a_{m}\right|=\left|f_{a}\left(e^{(m)}\right)\right| \leq\left\|f_{a} \mid\right\| e^{(m)}\left\|_{\mathscr{C}(\Lambda)} \leq\right\| f_{a} \| \frac{1}{s(1-t)} \quad \forall m \in \mathbb{N}_{0},
$$

and so $a \in l_{\infty}$. Further, $x \in \mathscr{C}(\Lambda)$ implies that $R_{n}(x / \Lambda) \in c s$ for all $n$, and $\Lambda R(x / \Lambda) \in$ $c_{0}$. Therefore $a \Lambda R(x / \Lambda) \in c_{0}$. Now (4.3) yields

$$
\sum_{n=0}^{\infty} a_{n} x_{n}=\sum_{n=0}^{\infty} R_{n}(x / \Lambda)(\Delta(\Lambda a))_{n} \quad \forall x \in \mathscr{C}(\Lambda) .
$$

Thus $R(x / \Lambda) \Delta(\Lambda a) \in c s$ for all $x \in \mathscr{C}(\Lambda)$. Now $x \in \mathscr{C}(\Lambda)$ if and only if $R(x / \Lambda) \in \mathscr{W}(\Lambda)$ and, by [2, Theorem 4], $\Delta(\Lambda a) \in \mathcal{W}^{\beta}(\Lambda)=w_{\infty}(\Lambda)$. But this means that $a \in c_{\infty}(\Lambda)$. Thus we have shown that $\mathfrak{C}^{\beta}(\Lambda) \subset c_{\infty}(\Lambda)$.

Now we show

$$
\|a\|_{\mathscr{C}(\Lambda)}^{*}=\|a\|_{\tilde{C}_{\infty}(\Lambda)} \quad \forall a \in \mathscr{C}^{\beta}(\Lambda) .
$$

Let $a \in \mathscr{C}^{\beta}(\Lambda)=c_{\infty}(\Lambda)$, by what we have just shown. Then by (4.9), for all $x \in \mathscr{C}(\Lambda)$,

$$
\begin{aligned}
\left|\sum_{n=0}^{\infty} a_{n} x_{n}\right| & \leq \sum_{v=0}^{\infty} \lambda_{n(v+1)} \max _{v}\left|R_{n}(x / \Lambda)\right| \frac{1}{\lambda_{n(v+1)}} \sum_{v}\left|(\Delta(\Lambda a))_{n}\right| \\
& \leq\|a\|_{\tilde{c}_{\infty}(\Lambda)}\|x\|_{\mathscr{C}(\Lambda)},
\end{aligned}
$$

and so

$$
\|a\|_{\mathscr{C}(\Lambda)}^{*} \leq\|a\|_{\tilde{c}_{\infty}(\Lambda)}
$$

Let $v_{m} \in \mathbb{N}_{0}$. By $v_{0, m}$, we denote the smallest integer with $0 \leq v_{0, m} \leq v_{m}$ for which

$$
\frac{1}{\lambda_{n\left(v_{0, m}+1\right)}} \sum_{v_{0, m}}\left|(\Delta(\Lambda a))_{n}\right|=\max _{0 \leq v \leq v_{m}}\left(\frac{1}{\lambda_{n(v+1)}} \sum_{v}\left|(\Delta(\Lambda a))_{n}\right|\right) .
$$

We define the sequences $R^{(m)}$ and $x^{(m)}$ by

$$
R_{n}^{(m)}= \begin{cases}\frac{1}{\lambda_{n\left(v_{0, m}+1\right)}} \operatorname{sgn}\left((\Delta(\Lambda a))_{n}\right) & \text { for } n \in N^{\left\langle v_{0, m}\right\rangle}, \\ 0 & \text { for } n \notin N^{\left\langle v_{0, m}\right\rangle},\end{cases}
$$


and $x_{n}^{(m)}=R_{n}^{(m)}-R_{n+1}^{(m)}(n=0,1, \ldots)$. Then we have

$$
\begin{gathered}
|| x^{(m)} \|_{\mathscr{C}(\Lambda)}=\sum_{v=0}^{\infty} \lambda_{n(v+1)} \max _{v}\left|R_{n}^{(m)}\right|=\lambda_{n\left(v_{0, m}+1\right)} \max _{v_{0, m}}\left|R_{n}^{(m)}\right| \leq 1, \\
\left|\sum_{n=0}^{\infty} a_{n} x_{n}^{(m)}\right|=\max _{0 \leq \nu \leq v_{m}} \frac{1}{\lambda_{n(v+1)}} \sum_{v}\left|(\Delta(\Lambda a))_{n}\right| \leq\|a\|_{\mathscr{C}(\Lambda)}^{*}\|x\|_{\mathscr{C}(\Lambda)} \leq\|a\|_{\mathscr{C}(\Lambda)}^{*} .
\end{gathered}
$$

Since $v_{m}$ was arbitrary, we obtain $\|a\|_{\tilde{c}_{\infty}(\Lambda)} \leq\|a\|_{\mathscr{C}(\Lambda)}^{*}$. Together with (4.12), this yields (4.10).

(c) Since $\mathscr{C}(\Lambda)$ has AK by part (b) and so AD, part (c) follows from [4, Theorem 7.2.7(ii) and (iii), page 106].

\section{REFERENCES}

[1] A. M. Jarrah and E. Malkowsky, The dual spaces of $\Lambda$-strongly convergent and bounded sequences, to appear.

[2] E. Malkowsky, Matrix Transformations in a New Class of Sequences that Includes Spaces of Absolutely and Strongly Summable Sequences, Habilitationsschrift, Giessen, Univ. Giessen, 1989. Zbl 684.46011.

[3] _ The continuous duals of the spaces $c_{0}(\Lambda)$ and $c(\Lambda)$ for exponentially bounded sequences $\Lambda$, Acta Sci. Math. (Szeged) 61 (1995), no. 1-4, 241-250. MR 97d:46005. Zbl 846.40008.

[4] A. Wilansky, Summability through Functional Analysis, North-Holland Mathematics Studies, 85, North-Holland Publishing Co., Amsterdam-New York, 1984, Notas de Matematica [Mathematical Notes], 91. MR 85d:40006. Zbl 531.40008.

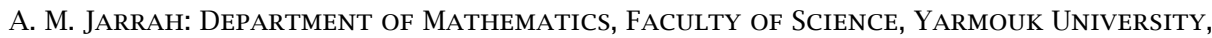
IRBID, JORDAN

E-mail address: ajarrah@yu.edu.jo

E. MALKowsky: C/O Department of Mathematics, FACUlty of SCIENCE, YARMOUK UNIVERSITY, IRBID, JORDAN

E-mail address: ma 7 kowsky@math. uni-giessen.de 


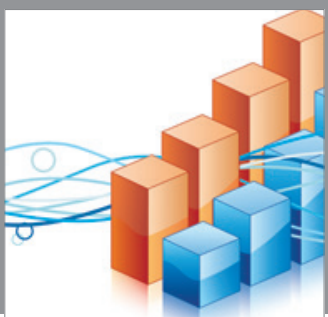

Advances in

Operations Research

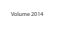

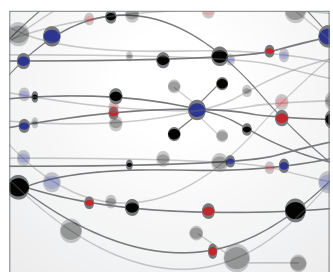

\section{The Scientific} World Journal
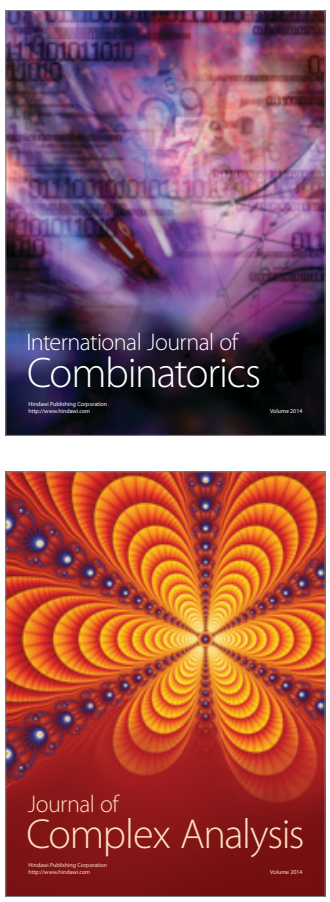

International Journal of

Mathematics and

Mathematical

Sciences
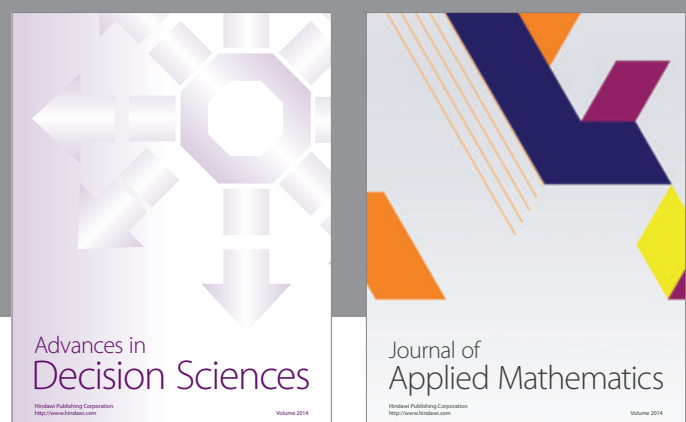

Journal of

Applied Mathematics
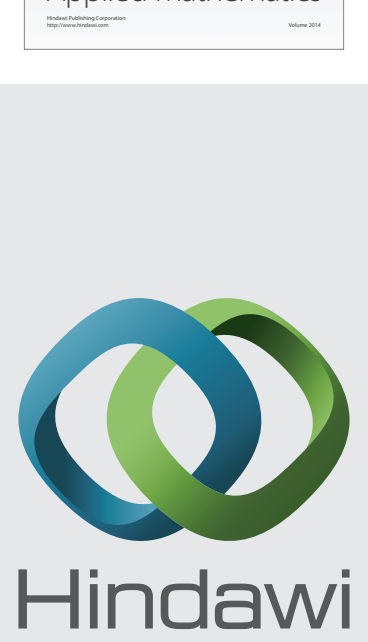

Submit your manuscripts at http://www.hindawi.com
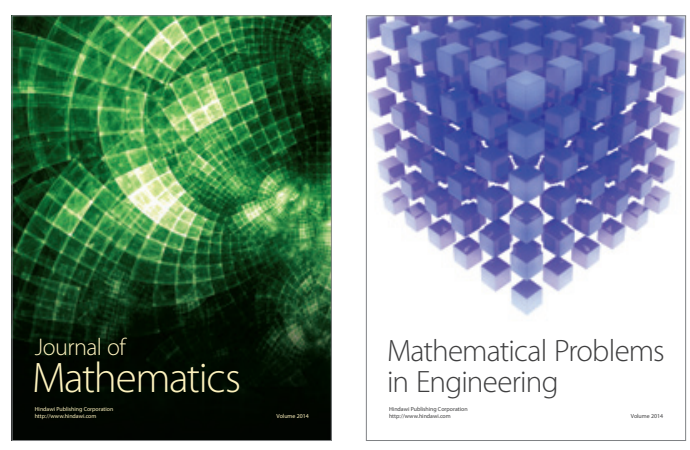

Mathematical Problems in Engineering
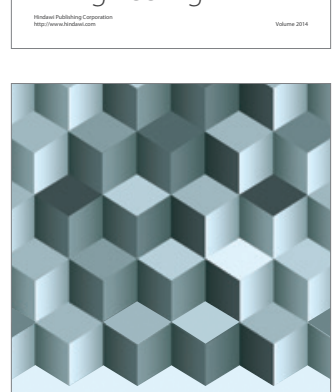

Journal of

Function Spaces
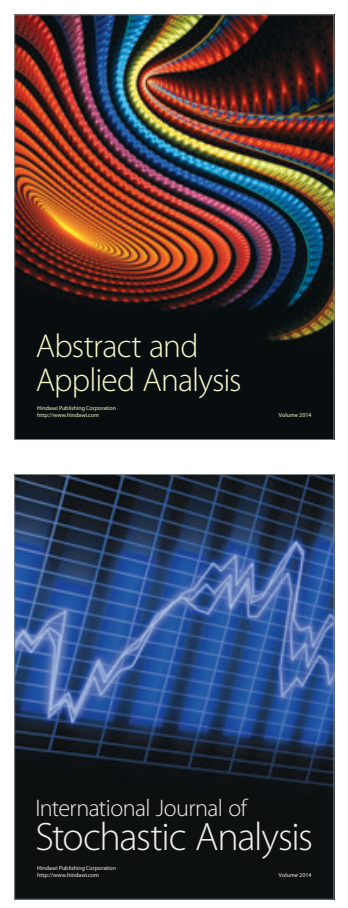

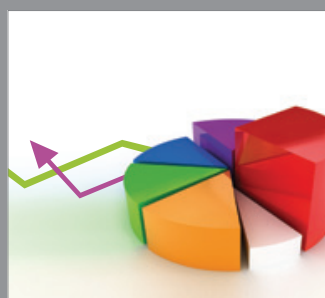

ournal of

Probability and Statistics

Promensencen
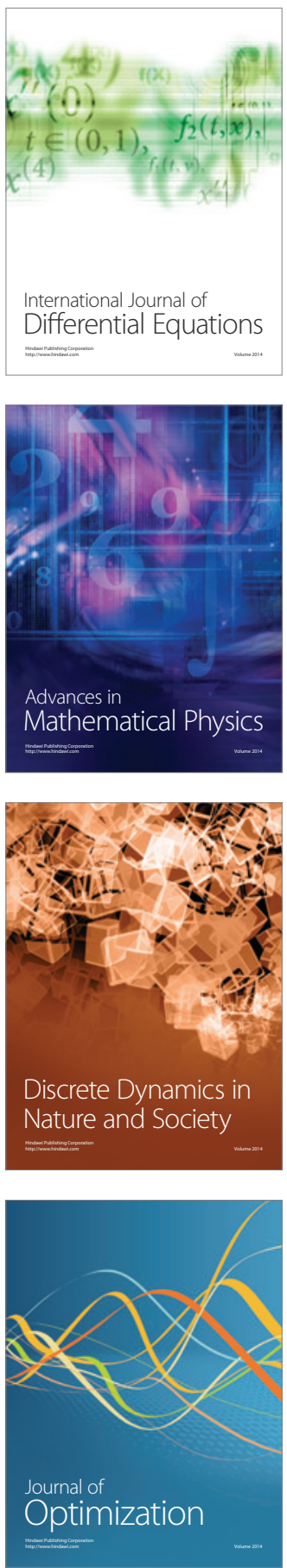